\title{
DIE 10-MINUTEN-SPRECHSTUNDE
}

\author{
Pralle Harnblase, doch es kommt fast kein Urin
}

Was tun bei schwachem Harnstrahl?

W. BRUMMEISL

\author{
Ein wohlstandsgenährter 67-Jähriger Patient kommt mit \\ stärksten Unterbauchschmerzen zu Ihnen in die Praxis. Er \\ berichtet, seit vier Stunden nicht mehr Wasserlassen zu können. \\ Im Ultraschall erkennen Sie in beiden Nieren einen Harnstau \\ und eine mit über $1000 \mathrm{ml}$ prall gefüllte Harnblase.
}

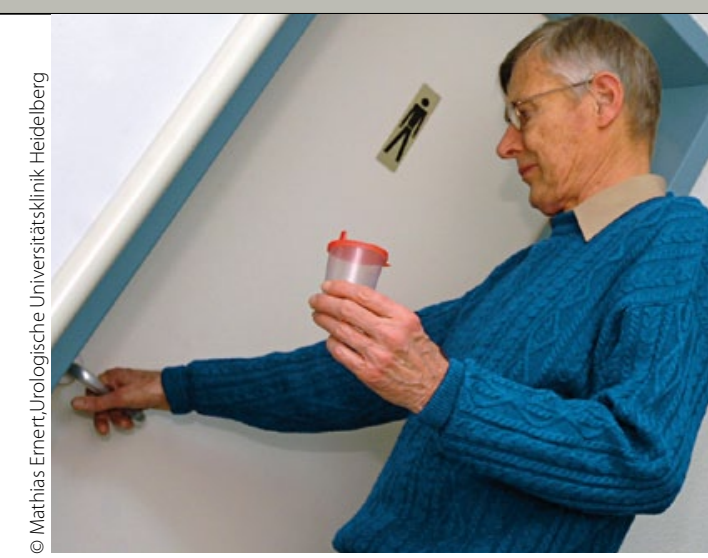

Zur Diagnostik gehört beim schwachen Harnstrahl auch ein Urinstatus.
— Mit der Verdachtsdiagnose eines akuten Harnverhaltes legen Sie einen Blasenkatheter 14 Charrière an, worauf sich klarer Urin entleert und die Symptomatik rasch abnimmt. Bei der Anamnese berichtet der Patient, dies sei das erste Mal passiert, jedoch habe er in letzter Zeit zunehmendes Pressen beim Wasserlassen und einen schwächer werdenden Harnstrahl bemerkt.

Ein schwacher Harnstrahl kann mehrere Ursachen haben. Bei Männern ab dem 50. Lebensjahr ist die Benigne Prostatavergrößerung ( $\mathrm{BPH})$ die häufigste Ursache von Beschwerden im Sinne eines LUTS (lower urinary truct syndrom). Unter LUTS fallen Symptome wie eine Harnstrahlabschwächung, verzögerter Miktionsbeginn, Nachträufeln, Pollakisurie, Nykturie, ein schmerzhafter Harndrang, Restharngefühl oder auch eine Dranginkontinenz.

\section{Was müssen Sie abklären?}

Trotz dieses oft eindeutigen Krankheitsbildes ist das Ziel der Diagnostik, die nicht $\mathrm{BPH}$ bedingten Ursachen eines LUTS auszuschließen. Darunter fallen u. a. das Prostatakarzinom, Harnröhrenstrikturen, Blasenauslassengen oder neurogene Blasenstörungen. Folgende Basisdiagnostik kann einfach erfolgen:

- Anamnese: ausführliche Miktionsanamnese, Nykturie, B-Symptome, Medikamenteneinnahme (Psychopharmaka, An- ticholinergika), Vorerkrankungen (Diabetes mellitus, Apoplexie, M. Parkinson) - Standardisierte Fragebögen: International Prostate Symptom Score (IPSS, Werte von 0-35), „quality of life“

. Körperliche Untersuchung: Nierenlager- oder Blasenschmerz

- Digital rektale Untersuchung: Größe, Konsistenz, Symmetrie (Memo: Die Größe der Prostata korreliert nicht mit der Symptomatik!)

- Harnstatus: Harnwegsinfekt (HWI), Mikrohämaturie

- Labor: Infekt, Kreatininerhöhung

- PSA: Normwert 2,5-4,5 ng/ml

- Ultraschall: Restharn, Hydronephrose.

\section{Was können Sie unternehmen?}

Wurde die Diagnose BPH gestellt, ist das Therapieziel, den Leidensdruck zu senken, einen Progress zu verlangsamen und v. a. Komplikationen zu vermeiden. Bei Beschwerdefreiheit oder leichter Symptomatik stehen zunächst folgende Therapiemaßnahmen zur Verfügung:

- Kontrolliertes Zuwarten: Lifestyleänderungen empfehlen, halb- bis jährliche Progressionskontrollen

- Phytopharmaka: Brennnesselwurzel, Sägezahnpalme (Memo: jedoch wenig wissenschaftlich fundiert, keine generelle Empfehlung)

- Pharmaka:

- a-Blocker (z. B. Tamsulosin 0,4 mg 1-0-0 p.o.): Wirkeintritt innerhalb von ein bis zwei Tagen, Wirkdauer ca. vier bis sechs Jahre.

- 5a-Reduktase-Hemmer (z. B. Finasterid 5 mg 1-0-0 p.o.): Wirkeintritt nach drei bis sechs Monaten, indiziert bei Prostatavolumen $>40 \mathrm{ml}$.

\section{Wann beim Urologen vorstellen?}

Falls die Beschwerden für den Patienten subjektiv belastend sind, Restharnmenge $>100 \mathrm{ml}$, eine der genannten Komplikationen wie z. B. HWI auftreten oder der V.a. eine andere Ursache der LUTSSymptomatik besteht, empfiehlt es sich, den Patienten zur weiteren Abklärung und Therapie urologisch vorzustellen.

\section{An was noch denken?}

Abschließend ist es wichtig, dem Patienten zu vermitteln, dass diese Erkrankung einer Progression unterliegt und regelmäßige Kontrollen notwendig sind. Es ist ausdrücklich darauf hinzuweisen, dass eine Operation der $\mathrm{BPH}$ nicht vor einem Prostata-Ca. schützt und Vorsorgeuntersuchungen einmal jährlich $\mathrm{ab}$ dem 45. Lebensjahr eingehalten werden sollten.

\section{Anschrift des Verfassers:}

Dr. med. Wolfgang Brummeisl

Klinik für Urologie - Lehrstuhl der Universität

Regensburg, Caritas-Krankenhaus St. Josef Landshuter Straße 65, D-93053 Regensburg E-Mail:Wolfgang.Brummeisl@klinik.uniregensburg.de 